\title{
Acesso ao cuidado na Atenção Primária à Saúde brasileira: situação, problemas e estratégias de superação
}

\author{
Access to care in Primary Health Care in Brazil: situation, problems \\ and coping strategies
}

Charles Dalcanale Tesser ${ }^{\mathbf{1}}$, Armando Henrique Norman², Tiago Barra Vidal3.

DOI: $10.1590 / 0103-110420185125$

1 Universidade Federal de Santa Catarina (UFSC)

Departamento de Saúde Pública - Florianópolis

(SC), Brasil.

Orcid: https://orcid. org/0000-0003-06508289

charles.tesser@ufsc.br

2 Universidade Federal de Santa Catarina (UFSC),

Departamento de Clínica Médica - Florianópolis

(SC), Brasil.

Orcid: https://orcid. org/0000-0002-5267-

7377

a.h.norman@ufsc.br

3 Universidade Federal de Santa Catarina (UFSC) -

Florianópolis (SC), Brasil. Orcid: https://orcid. org/0000-0002-61039625

tbvidal@gmail.com
RESUMO Sistemas de saúde universais orientados pela Atenção Primária à Saúde (APS) apresentam melhores resultados para a população. Este artigo apresenta a situação do acesso ao cuidado na APS brasileira, seus problemas, desafios e estratégias para sua superação. Realizou-se uma revisão narrativa, incluindo estudos quali e quantitativos. O acesso na APS aumentou com a expansão da Estratégia Saúde da Família (ESF), mas ainda permanece insuficiente. As principais barreiras ao acesso incluem: subdimensionamento/subfinanciamento da APS, excesso de usuários vinculados às equipes da ESF, número reduzido de Médicos de Família e Comunidade (MFC), com pouca interiorização/fixação, burocratização e problemas funcionais dos serviços, como rigidez nos agendamentos e priorização de grupos específicos (hipertensos, puericultura etc.). Para melhorar o acesso, é necessário aumentar o investimento federal na ESF, priorizando-a e expandindo-a, reduzir os usuários vinculados às equipes, ampliar a formação médica em MFC, explorar a clínica da enfermagem, diversificar os meios de comunicação com usuários, explorar a cogestão da equipe e flexibilizar as agendas dos profissionais. Conclui-se que, para fortalecer a APS, é estratégico estimular o acesso na ESF vinculado ao cuidado longitudinal.

PALAVRAS-CHAVE Atenção Primária à Saúde. Acesso aos serviços de saúde. Sistema Único de Saúde.

ABSTRACT Universal health systems oriented by Primary Health Care (PHC) present better population outcomes. This article addresses the situation of access to care in Brazilian PHC. A non-systematic narrative review was carried out, which included published studies and empirical experiences in several services and municipalities. Three thematic axes guide the present study: (1) Current access situation in Brazilian PHC; (2) Problems and challenges; and (3) Strategies for expanding access in PHC. Research shows that access to PHC increased with the expansion of the Family Health Strategy (FHS), but still remain insufficient. The main barriers to access include: undersizing and underfunding of PHC, excessive patient-list linked to FHS teams, reduced number of specialist physicians, difficulty in interiorizing/fixing FHS professionals, and excessive municipal autonomy in the management of PHC services. To improve and qualify the access in PHC, it is necessary to increase federal investments, expand FHS coverage, reduce patient-list linked to FHS teams, 
increase medical training in family and community medicine, optimize nursing clinics, diversify communication media with users, and enhance appointment flexibility. In conclusion, to strengthen PHC it is strategical to stimulate access to longitudinal care.

KEYWORDS Primary Health Care. Health services accessibility. Unified Health System.

\section{Introdução}

Há 40 anos, a Declaração de Alma Ata' estimulou a expansão da Atenção Primária à Saúde (APS) no mundo. A APS requer melhorias sociais amplas. Pressupõe a indissociabilidade entre saúde e desenvolvimento social, com ação intersetorial para enfrentar os determinantes sociais da saúde-doença (entre eles, a distribuição das riquezas é muito importante), maior democratização e participação social e o acesso universal a uma rede de serviços de assistência à saúde ${ }^{2,3}$, doravante chamados Centros de Saúde (CS). Estes realizam o primeiro contato dos cidadãos com o sistema de saúde. Os profissionais dos CS são geralmente generalistas, resolvem a grande maioria dos problemas de saúde da população e exercem a função de 'filtro' (gatekeeper) ${ }^{4}$, regulando e coordenando o acesso não emergencial dos usuários a outros serviços do sistema.

Há robustas evidências sobre as vantagens de sistemas de saúde baseados em APS: melhores indicadores de saúde ${ }^{5-8}$, especialmente infantis ${ }^{5}$; redução dos anos potenciais de vida perdidos ${ }^{6}$; maior acesso e qualidade assistencial a serviços de saúde 7,8; melhor desempenho na prevenção das doenças e promoção da saúde ${ }^{8}$; melhor desempenho dos sistemas de saúde, com menos gastos e menos internações por vários problemas ${ }^{6-15}$; melhoria dos níveis de saúde das populações ${ }^{6-8,13,16}$ e redução das iniquidades em saúde ${ }^{8,9}$.

No Sistema Único de Saúde (SUS), a APS ou atenção básica está presente na legislação do SUS contida na diretriz 'hierarquização'. As normativas atuais convergem para seu papel fundamental de porta de entrada principal e coordenadora da rede de atenção à saúde ${ }^{17,18}$. A descentralização/municipalização do SUS conferiu autonomia aos municípios na gestão dos serviços de APS, gerando grande heterogeneidade desses serviços ${ }^{19,20}$.

A criação do Programa Saúde da Família (PSF), depois Estratégia Saúde da Família (ESF), significou uma indução financeira federal exitosa de ampliação e organização de equipes de APS. Com a ESF, equipes com médicos, enfermagem e Agentes Comunitários de Saúde (ACS) (opcionalmente dentistas/auxiliares) passaram a realizar trabalho clínico, preventivo e promocional, vinculadas a usuários territorialmente definidos. A ESF expandiu-se inicialmente mais no interior e em pequenas cidades. As grandes cidades e capitais (salvo exceções) implantaram mais tardiamente a ESF e converteram menos seus CS à ESF 21. Mesmo assim, a ESF expandiu-se e transformou-se no principal formato brasileiro de equipes de APS, mas não viabilizou uma cobertura universal, como se verá adiante.

Não há dúvida sobre a superioridade da ESF em relação aos CS sem ela. Em 2012, 
revisão de Mendes $^{22}$ não encontrou nenhum estudo evidenciando benefício para o modelo tradicional em relação à ESF. Igualmente, nenhuma pesquisa abordada neste artigo (ou de que temos ciência) mostrou vantagens dos CS sem ESF em relação aos com ESF, o que converge com a sistematização de evidências de Fachini et al. ${ }^{23} \mathrm{em} 2014$. A ESF tem melhor desempenho quanto à integralidade, orientação comunitária e familiar ${ }^{24}$, acesso ${ }^{25} \mathrm{e}$ utilização dos serviços ${ }^{26,27}$; maior satisfação de usuários e profissionais $\mathbf{2 8}^{\mathbf{2 9}}$; maior equidade ${ }^{30,31}$; maior utilização de atendimento domiciliar ${ }^{32,33}$; melhor consciência da população sobre as doenças crônicas ${ }^{\mathbf{3 4}}$; melhor assistência materno-infantil35,37. Ela está mais presente em locais mais afastados, pobres e com pior infraestrutura. Sua capilaridade facilita o acesso da população socialmente vulnerável ${ }^{30}$. A ESF melhorou o desempenho do SUS e as condições de saúde ${ }^{22}$ : redução da mortalidade infantil ${ }^{\mathbf{3 8}-40}$, da mortalidade adulta $^{\mathbf{4 1}}$, associou-se a aumento da frequência escolar e da oferta de trabalho e emprego em adultos ${ }^{40}$, a melhorias na qualidade das informações sobre as causas de morte ${ }^{42} \mathrm{e}$ à diminuição das hospitalizações por condições sensíveis à APS ${ }^{43-47}$.

Para a APS prover e coordenar efetivamente um cuidado longitudinal, abrangente (integral) e orientado à família e comunidade, o acesso ao cuidado é condição si ne qua non $^{\mathbf{4 8}}$. No Brasil, apenas a ESF aproxima-se disso, mas a APS nunca teve regra clara para a provisão de acesso oportuno, em escala nacional ou em contextos locais ${ }^{49}$.

Diante do exposto, o objetivo deste artigo é apresentar um panorama sintético da situação do acesso ao cuidado na APS, apontar problemas e desafios e sugerir estratégias para sua superação. Realizou-se uma revisão narrativa assistemática que incluiu estudos quali e quantitativos. Devido à ausência de dados sistematizados nacionais sobre acesso na APS, foram considerados estudos locais e enfatizados aspectos organizacionais mais estudados no País.
Para facilitar a apresentação, não foi feita a distinção proposta por Starfield ${ }^{50}$ entre acesso e acessibilidade, cujos significados foram englobados no termo 'acesso'. Tampouco esgotamos as várias dimensões do acesso (geográfica, política, econômico-social, técnica, organizacional e simbólica, conforme Assis e Jesus ${ }^{51}$ ), embora nossas proposições transpassem várias delas.

\section{Acesso na APS: situação atual}

Na conformação atual do acesso na APS, uma diretriz nacional merece menção: o 'acolhimento'52,53. Nascido em contexto de comum degradação da relação entre serviços e usuários, associado à precariedade do acesso, o acolhimento visa resgatar a relação de solidariedade profissional-usuário e a agilidade do acesso na APS. Ele orienta a prática de escuta empática de todos os usuários nos serviços de APS, com negociação entre profissionais, visando aumentar a equidade, a resolubilidade do primeiro contato e a agilidade no atendimento. $\mathrm{O}$ acolhimento tem estimulado a prática clínica da enfermagem e o trabalho em equipe, a desburocratização organizacional e a melhoria do acesso ${ }^{54-56}$.

Quanto à situação atual do acolhimento, todavia, a ausência de articulação em redes integradas, o excesso de demanda, a ausência de capacitação e de espaços democráticos para reorganizar o processo de trabalho têm colocado em questão a potencialidade dessa diretriz ${ }^{54}$. Há grande heterogeneidade de práticas sob essa designação, desde distribuição de fichas ou senhas para organizar a fila ${ }^{57}$, incluindo seleção de quem vai preencher as vagas destinadas à 'demanda espontânea' nas agendas, até conversa coletiva da equipe com usuários na primeira hora do turno, seguida de negociações individuais e atendimentos clínicos ${ }^{58}$.

Quanto à cobertura da APS brasileira, a mais recente Pesquisa Nacional de Saúde ${ }^{59}$ 
mostrou que $72 \%$ da população não tem planos de saúde privados; $56 \%$ desta está cadastrada em uma equipe de saúde da família; $60 \%$ dos respondentes que foram atendidos em algum serviço ambulatorial, o foram no SUS; e mais na APS em relação às pesquisas anteriores. Os dados do Ministério da Saúde são convergentes: estimando 3 mil usuários por equipe de saúde da família, as 42.855 equipes implantadas até 05 de abril de $2018^{60}$ cobrem $62,4 \%$ da população brasileira, sem menção aos CS sem ESF. Embora não se saiba que proporção da população atendida pela APS é atendida na ESF, Bousquat et al.61, a partir de dados do censo de unidades básicas de saúde do Programa Nacional de Melhoria do Acesso e da Qualidade da Atenção - PMAQ-AB - de 2012 realizado, que computou 38.812 unidades, em 5.543 municípios, identificaram que em $77 \%$ deles havia alguma equipe da ESF com ou sem equipe de saúde bucal.

Várias pesquisas locais foram publicadas sobre acesso/acolhimento, com métodos qualitativos e quantitativos. Embora a ESF tenha ampliado o acesso ${ }^{62-64}$, há consenso de que ele ainda é insuficiente no País ${ }^{65}$, mesmo em locais onde a ESF já está estabeleci$\mathrm{da}^{66-68}$. As pesquisas qualitativas mostram dificuldade no acesso, com tempo de espera para atendimento demorado, levando uma ou várias semanas para a maioria dos serviços $65,66,69-71$, com persistentes problemas de acolhimento na sua dimensão relacional72,73.

Os estudos quantitativos também convergem. A maioria deles utiliza o Primary Care Assessment Tool (PCATool). O PCATool é internacionalmente reconhecido como instrumento que mensura os atributos da APS - acesso, integralidade, longitudinalidade, coordenação do cuidado, abordagem familiar e comunitária e competência cultural ${ }^{50}$ - e foi validado para a realidade brasileira ${ }^{76}$. Avaliado pelo PCATool em vários locais, o acesso é o atributo da APS com pior avaliação, na ESF e fora dela ${ }^{68,75-81}$, sendo geralmente superior na ESF em relação aos CS sem ESF 82,83, e melhor em alguns locais do Sudeste 55,71 . No PCATool, o atributo 'acesso de primeiro contato' possui duas subdimensões avaliadas: acessibilidade e utilização. Os baixos escores do acesso se dão principalmente na subdimensão acessibilidade, composta por várias perguntas relativas à facilidade de obtenção de, por exemplo: atendimento no mesmo dia em caso de adoecimento; aconselhamento rápido por telefone se precisar; agendamento de uma consulta de revisão; agendamento de uma consulta no serviço etc. Essa subdimensão identifica se o serviço de saúde é facilmente acessível e disponível.

Como síntese geral, as pesquisas disponíveis indicam que o acesso é ruim, e isso se reflete nos demais atributos da APS e na efetividade do SUS.

\section{Problemas e desafios}

De acordo com Conill184, o acesso permanece como um nó crítico da APS brasileira, com vários problemas associados comprometendo-o. Há concordância nas pesquisas quanto à excessiva população vinculada às equipes da ESF, piorando diretamente o acesso. Há grande centralização no atendimento médico, embora Campos et al. ${ }^{85}$ apontem que nenhum sistema universal de saúde do mundo funciona sem garantir, com agilidade, o acesso ao médico generalista. Por exemplo, o National Health Service britânico provê a seus usuários acesso a um profissional médico em no máximo dois dias úteis ${ }^{86}$.

Um problema comumente envolvido na precariedade do acesso é a priorização de grupos considerados preferenciais: grávidas, hipertensos, puericultura etc. A ênfase na prevenção da APS brasileira se associa com a virada preventivista da medicina contemporânea, gerando iniquidades e acirrando problemas de acesso ${ }^{\mathbf{4 8}, 49,87-89}$. Tal ênfase induz excesso e ou precária fundamentação de atividades preventivas individuais, que 
têm alto potencial de danos iatrogênicos e sobremedicalização do cuidado ${ }^{90}$, como rastreamentos de câncer de colo de útero anuais e de câncer de próstata em qualquer idade ${ }^{\mathbf{9}}$, rastreamentos anuais de câncer de mama (e em menores de 50 anos em qualquer periodicidade) ${ }^{\mathbf{9 2}}$, exames de sangue urina e fezes de rotina em crianças $^{93}$, consultas médicas de rotina ${ }^{94}$ etc.

Outro problema da APS brasileira é a sua burocratização, traduzida em horários restritos, prejudicando os usuários que trabalham e que poderiam utilizar os serviços no horário de almoço ou após as 17 horas ${ }^{95}$; profissionais administrativos nas recepções com pouca competência comunicativa; pouca agilidade administrativa ante as atuais tecnologias, como telefone, correio eletrônico e comunicadores digitais, exigindo-se a presença dos usuários para obter qualquer informação/ orientação ou resolver qualquer demanda ${ }^{56,66}$.

Os problemas acima apontados geram uma cultura institucional e rotinas de contato usuário-equipe apenas presenciais, que sustentam recepções problemáticas, rígidas e pouco adaptáveis às necessidades dos usuários, criando comumente um 'fechamento' dos serviços, proteção dos profissionais do excesso de demanda e precarização do acesso.

Quanto ao acolhimento, tem sido percebido pelos profissionais como mais uma tarefa entre outras. Tende a causar sobrecarga de trabalho, cansaço, estresse e conflito nas equipes, sobretudo quando ações de ampliação de acesso são desenvolvidas ${ }^{56}$. As dificuldades para sua operacionalização incluem: excessivo número de usuários vinculados às equipes da ESF; falta de ambiência, de recursos básicos, infraestrutura e de formação para o trabalho na APS; insuficiência de profissionais e poucos espaços para discussões e fortalecimento do trabalho em equipe ${ }^{96,97}$. $O$ acolhimento não é de fácil operacionalização e foi considerado uma tecnologia assistencial incipiente por Sisson et al. ${ }^{70}$, se é que deve ser assim considerado ${ }^{49}$. De todo modo, o acolhimento é sinérgico com a ampliação do acesso, destacando que deve ser ágil e altamente empático. Bem trabalhado, o acolhimento aumenta a reciprocidade e harmonia na equipe ${ }^{\mathbf{9 8}}$, necessários para um bom acesso.

A escassez de médicos na APS com formação em Medicina de Família e Comunidade (MFC), sua concentração em grandes áreas urbanas e a sua grande rotatividade nos serviços indiretamente prejudicam o acesso (pela falta de profissionais e pelo não conhecimento dos usuários, tornando os atendimentos mais trabalhosos). Havia, em 2017, 5.438 mil médicos de família e comunidade no Brasil99, 3.341 com residência em MFC e o restante somente com título de especialista; o que é pouco mais de $10 \%$ dos médicos da ESF. Os com residência em MFC são 7,8\% dos médicos da ESF, e menos de $1 \%$ dos 452.801 médicos do País em 2018 ${ }^{100}$. Em comparação grosseira, na Inglaterra, havia, em 2009, quase 0,8 generalistas (General Practitioners ou GP) por mil habitantes ${ }^{\mathbf{1 0 1}}$. Supondo os GP equivalentes aos MFC brasileiros, o Brasil tem aproximadamente $0,026 \mathrm{MFC}$ por mil habitantes (5.438/209 milhões), proporcionalmente 31 vezes menos médicos com titulação em MFC e 50 vezes menos médicos com residência específica para atuação na APS.

O financiamento federal da APS é um grande problema, pois o SUS não tem recebido suficiente aporte financeiro federal para alcançar a universalidade ${ }^{\mathbf{1 0 2}}$. Em relação ao financiamento da APS, a participação proporcional dos gastos federais vem diminuindo, enquanto o gasto municipal, vinculado à APS, vem aumentando ${ }^{103}$.

\section{Estratégias para ampliação do acesso na APS}

Diante da precariedade do acesso e do subdimensionamento da rede de serviços de APS, várias estratégias associadas se impõem para sua melhoria, abordadas sinteticamente a seguir.

'Aumento de cobertura e redução da 
população vinculada às equipes da ESF'. A ESF deve ser priorizada como o formato da APS brasileira, por ser o melhor modelo testado nacionalmente e o que mais facilitou a concretização dos atributos da APS. Infelizmente, as recentes mudanças ocorridas na Política Nacional da Atenção Básica (PNAB) de 2017 diminuíram a importância da ESF no conjunto da APS104. É necessário ampliar a cobertura da APS via ESF e diminuir a população vinculada a cada equipe. Estimando que $25 \%$ da população adulta adoece e procura ajuda profissional em um mês ${ }^{\mathbf{1 0 5}}$, e que um médico atende a aproximadamente 500 pessoas por mês, o que significa mais de 500 consultas (com a enfermeira fazendo uma parte desse atendimento), os ingleses determinam como máximo de pessoas vinculados a um generalista $2 \mathrm{mil}$ usuários, sendo a média menor que isso ${ }^{49}$. Quase todos os países com APS estruturada acompanham esse padrão ${ }^{106,107}$.

'Conversão para a ESF'. A consensual superioridade da ESF demanda priorizar a conversão progressiva dos CS sem ESF para ela. Isso demanda investimento federal tanto em normativas pró-ESF quanto em recursos financeiros, que podem induzir a conversão, ao contrário da direção da PNAB de 2017. Dada a concentração tributária na União, é imprescindível que esta aumente os repasses para os municípios privilegiando a ESF. Uma estratégia possível de facilitação da conversão para a ESF seria a indução da construção de vínculo longitudinal equipes-usuários nos CS sem ESF. Por exemplo, esses serviços poderiam ser adaptados por meio de listas de usuários vinculados a equipes. Outra estratégia possível para reforçar a ESF onde ela já existe seria permitir alguma escolha para o usuário, se ele desejar, de sua equipe, quando há outras equipes atuando no mesmo CS, por meio de listas de usuários associadas com vinculação territorial. Isso compensaria parcialmente a restrição de liberdade de escolha implícita na função filtro e, em tese, reforçaria a longitudinalidade, devido a um melhor (e 'mais' voluntário) vínculo terapêutico ${ }^{108}$.

'Interiorização e fixação de profissionais (sobretudo médicos)' é um ponto estratégico. O Programa Mais Médicos ${ }^{109}$ tem sido a via de enfrentamento da pouca interiorização dos médicos na APS, sua alta rotatividade, escassez e pouca competência técnica, devido à falta de formação adequada em MFC. Apenas investimentos federais sérios e urgentes são capazes de abordar esse problema tanto por meio da ampliação das residências em MFC quanto pela estruturação de gestão regional intermunicipal. O pequeno número de programas de residência em $\mathrm{MFC}$, sua qualidade heterogênea e a pequena tradição da especialidade no País são problemas de grande vulto que necessitam ações em escala nacional, como iniciou o Mais Médicos. Essa estratégia, todavia, precisa ser aperfeiçoada e implantada na sua faceta de formação, com grande ampliação das vagas e programas de residência em MFC. A meta é exigir residência em MFC para os médicos da ESF para breve, estruturando a APS via ESF.

Para melhorar a equidade no acesso, deve-se priorizar áreas de vazio assistencial e maior vulnerabilidade social. Atualmente, quando existe, a priorização é intramunicipal e com ônus total para o município. Tal priorização pode ser de várias ordens e incluir inclusive estímulo financeiro aos profissionais, e poderia ser institucionalizada em regiões de saúde, para diminuir a grande heterogeneidade da APS ${ }^{19}$.

'Desburocratizar e ampliar o acesso via organização funcional das equipes'. Várias melhorias no funcionamento dos serviços são necessárias, sobretudo quanto aos problemas destacados acima, abordados a seguir.

'Horário de funcionamento'. O acesso é diretamente ampliado com a extensão do horário de funcionamento dos CS para o horário de almoço e noturno, bem como a utilização dos sábados ${ }^{95}$.

'Reuniões periódicas das equipes da ESF'. Para a ampliação do acesso, é estratégico o uso judicioso de tempos periódicos de 
conversa para organização, gestão e decisão coletivas na equipe: as reuniões (pelo menos) semanais. Elas são necessárias para gestão dos processos de trabalho implicados no acesso oportuno e para elaboração de projetos terapêuticos, gestão das consultas/visitas domiciliares, coordenação do cuidado para casos mais complexos etc. Gestão participativa das rotinas que interferem diretamente no acesso é fundamental para haver espírito de equipe, participação ativa e consciente e maior cooperação mútua entre profissionais ${ }^{110}$.

'Diversificar formas de comunicação dos usuários com a equipe' é estratégico para melhorar o acesso, incluindo uso de telefone (correio eletrônico e outros comunicadores), que deve se transformar em recurso cotidiano de atendimento, agendamento e orientação dos usuários. Tal diversificação facilita a comunicação equipe-usuários, melhora o vínculo e maximiza o acesso ${ }^{48,111}$. Isso exige o fortalecimento dos aspectos administrativos viabilizadores dessa diversificação. $\mathrm{O}$ reforço administrativo viabiliza o uso do telefone, correio eletrônico e comunicação digital, superando a necessidade de contato presencial para encaminhamento de qualquer demanda dos usuários.

'Otimização do papel da enfermagem'. Para ampliar o acesso e organizar o trabalho em equipe, é necessário o investimento no trabalho clínico da enfermagem, já induzido pela diretriz do acolhimento. A enfermagem tem grande potência clínica, geralmente subaproveitada ${ }^{112}$, sobretudo em casos crônicos e ações preventivas, que necessitam de grande empatia e competência educativa. Também tem grande capacidade de avaliar pequenas urgências/intercorrências, devendo ser parceiras na agilização do acesso.

'Viabilização de sistema de informações'. A disponibilização de prontuário eletrônico (e-SUS) e sistemas de informações facilita e viabiliza o acesso indiretamente. A história clínica é essencial para um acesso longitudinal adequado. Isso implica pesado investimento federal na melhoria e universalização do e-SUS.
'Diminuir a rigidez das agendas, tornando-as mais inteligentes'. Trata-se de aproximar-se da 'filosofia' do chamado acesso avançado: fazer o serviço de hoje, hoje ${ }^{113,114}$. Implícita nesta 'filosofia' está a superação necessária da dicotomia hierarquizada entre demanda espontânea e programada, em que há hipervalorização da segunda. Isso torna o serviço mais acessível e minimiza o empurrar para o futuro de ações clínicas e outras. A esse respeito, não raramente aparecem preocupações relativas à necessidade de cuidados continuados programados voltados às situações crônicas. Parece certo que a longitudinalidade associada ao acesso oportuno é uma das chaves para a efetividade e qualidade do cuidado a longo prazo. Isso reforça que o acesso deve estar vinculado à longitudinalidade. A necessária agilidade na comunicação com os usuários (acima referida) facilita atividades de vigilância nos problemas crônicos, que se tornam proeminentes com o envelhecimento populacional. Também, por isso, o primeiro contato sempre que possível deve ser realizado por um profissional da equipe vinculada ao usuário, melhorando sua resolubilidade.

'Melhorar a qualificação clínica dos profissionais'. As situações crônicas e a ampliação do acesso exigem melhor qualificação clínica dos profissionais. A maior multimorbidade e frequência de problemas crônicos dificultam o uso racional dos recursos diagnósticos e terapêuticos, facilitam a polifarmácia, a iatrogenia e a medicalização desnecessárias, gerando indiretamente dificuldades de $\operatorname{acesso}^{\mathbf{4 8}}$. A longitudinalidade funciona como antídoto a essa tendência, mas requer qualificação clínica e prevenção quaternária ${ }^{115}$, com maior uso de desprescrição ${ }^{116}$, de cuidados paliativos e de abordagens menos medicalizadoras. Quanto a esse aspecto, destacam-se os problemas de saúde mental, altamente prevalentes e relevantes na APS. Eles precisam de uma abordagem psicossocial ${ }^{117}$ coerente com a reforma psiquiátrica brasileira, ao invés da comum atenção centrada em psicofárma$\cos ^{118}$, cujo uso corriqueiro é excessivamente 
medicalizante, pouco efetivo e gera aumento crônico da demanda (por psicotrópicos), dificultando o acesso. As práticas integrativas e complementares podem ser exploradas na APS nos crônicos (muito usadas) e em saúde mental (coerentes com a atenção psicossocial, em que promoção da saúde e terapêutica comumente se confundem) $)^{\mathbf{1 1 8 , 1 1 9}}$, como ocorre no mundo ${ }^{\mathbf{1 2 0}}$.

'Minimizar intervenções preventivas individuais pouco fundamentadas': o excesso de atividades preventivas individuais na APS deve ser evitado, devido ao seu alto potencial de geração de dano, medicalização e aumento da demanda. Tal excesso converte assintomáticos em preocupados e doentes crônicos, gera consultas e exames de rotina (rastreamentos mal fundamentados repetidos) que requerem seguimento etc. ${ }^{121}$. Isso demanda clara diferenciação entre cuidado aos adoecidos e prevenção em assintomáticos ${ }^{122}$ e intensa prática de prevenção quaternária na prevenção, de modo a reduzir a sobrecarga assistencial desnecessária gerada pelo excesso de preventivismo.

'Estabelecer regras para garantia do acesso': o acesso pode e deve ser objeto de regramento e metas claras, por meio de acordos e pactuações regionais e intramunicipais entre gestores, profissionais e representantes dos usuários ${ }^{49}$. Podem ser estabelecidos padrões pactuados de acesso (tempo de espera, por exemplo), favorecendo seu monitoramento pela gestão local, pelos usuários e Conselhos Municipais de Saúde, o que deve estar associado com compromissos de manutenção de completude mínima nas equipes, viabilizadora dos padrões. Paradoxalmente, no Brasil, o acesso, atributo crucial da APS, não foi objeto de regramento ao que sabemos. Alguns serviços têm conseguido atingir padrões de acesso de boa qualidade, quase sem registro disponível na literatura científica. Nesses serviços, o atendimento dos usuários vinculados tem ocorrido em menos de uma semana, preferencialmente em dois a três dias ${ }^{\mathbf{1 2 3}}$.

\section{Considerações finais}

Os estudos disponíveis e a prática assistencial convergem para a constatação de que o acesso na APS brasileira é heterogêneo e permanece precário, envolvendo vários problemas anteriormente apontados. As estratégias principais para ampliação do acesso na APS para quase toda a população envolvem fortalecer e privilegiar a expansão da ESF associada à redução da população vinculada a cada equipe. É necessário maior financiamento federal para a ESF, forte investimento na formação de médicos de família e comunidade (e educação permanente das equipes atuais), estímulo à sua interiorização e fixação, sobretudo em áreas de maior vulnerabilidade.

Do ponto de vista organizacional nos serviços, é estratégica a exploração do trabalho em equipe, da potencialidade clínica da enfermagem, da cogestão dos processos de trabalho, da flexibilização das agendas e da diversificação das formas de comunicação entre equipe e usuários, de modo a superar a necessidade do contato presencial para encaminhamento de qualquer demanda. A APS deve praticar uma atenção à saúde abrangente que envolva igualmente situações agudas e crônicas, em um contínuo de cuidados ampliados centrados na pessoa, na família e na comunidade pela mesma equipe, com coordenação dos cuidados especializados cabíveis. $\mathrm{O}$ acesso é a pedra angular sem o qual esses atributos não são viabilizados.

\section{Colaboradores}

Tesser CD elaborou a concepção do artigo e o rascunho inicial. Norman AH e Vidal TB contribuíram significativamente na revisão crítica do conteúdo, atualização bibliográfica e redação das versões sucessivas do artigo. Todos aprovaram a versão final do manuscrito. 


\section{Referências}

1. Declaração de Alma-Ata. In: Conferência internacional sobre cuidados primários de saúde [internet]. Alma-Ata; 1978 [acesso em 2017 ago 7]. p. 3. Disponível em: http://cmdss2011.org/site/wp-content/ uploads/2011/07/Declaração-Alma-Ata.pdf.

2. Stringhini S, Carmeli C, Jokela M, et al. Socioeconomic status and the $25 \times 25$ risk factors as determinants of premature mortality: a multicohort study and meta-analysis of $1 \bullet 7$ million men and women. Lancet [internet]. 2017 [acesso em 2018 maio 28]; 389(10075):1229-37. Disponível em: http://www. ncbi.nlm.nih.gov/pubmed/28159391.

3. Aleixo JLM. Atenção Primária à Saúde e o Programa de Saúde da Família: perspectivas de desenvolvimento no início do terceiro milênio. Rev Min Saúde Pública [internet]. 2002 [acesso em 2018 out 14]; 1(1):1-16. Disponível em: http://coleciona-sus.bvs. br/lildbi/docsonline/get.php?id=391.

4. Gérvas J, Fernández MP. El fundamento científico de la función de filtro del médico general. Rev Bras Epidemiol [internet]. 2006 [acesso em 2018 maio 28]; 9(1):147-9. Disponível em: http://www.scielo. br/scielo.php?script=sci_arttext\&pid=S1415-790X2 006000100019\&lng=es\&tlng=es.

5. Starfield B. Primary care and health. A cross-national comparison. JAMA [internet]. [acesso em 2018 ago 9]; 266(16):2268-71. Disponível em: http://www. ncbi.nlm.nih.gov/pubmed/1920727.

6. Macinko J, Starfield B, Shi L. The contribution of primary care systems to health outcomes within Organization for Economic Cooperation and Development (OECD) countries, 1970-1998. Health Serv Res [internet]. 2003 [acesso em 2018 maio 28]; 38(3):831-65. Disponível em: http://www.ncbi.nlm. nih.gov/pubmed/12822915.

7. Starfield B. Is primary care essential? Lancet [internet]. 1994 [acesso em 2018 mar 10]; 344(8930):112933. Disponível em: http://linkinghub.elsevier.com/ retrieve/pii/S0140673694906343.
8. Starfield B, Shi L, Macinko J. Contribution of Primary Care to Health Systems and Health. Milbank Q [internet]. 2005 [acesso 2018 maio 28]; 83(3):457502. Disponível em: http://www.ncbi.nlm.nih.gov/ pubmed/16202000.

9. Atun R. What are the advantages and disadvantages of restructuring a health care system to be more focused on primary care services? [internet]. 2004 [acesso em 2018 maio 28]. Disponível em: http://www.euro.who.int/_data/assets/pdf file/0004/74704/E82997.pdf.

10. Casanova C, Starfield B. Hospitalizations of Children and Access to Primary Care: A Cross-National Comparison. Int J Heal Serv [internet]. 1995 [acesso em 2018 maio 28]; 25(2):283-94. Disponível em: http://www.ncbi.nlm.nih.gov/pubmed/7622319.

11. Forrest CB, Starfield B. The effect of first-contact care with primary care clinicians on ambulatory health care expenditures. J Fam Pract [internet]. 1996 [acesso em 2018 maio 28]; 43(1):40-8. Disponível em: http://www.ncbi.nlm.nih.gov/pubmed/8691179.

12. Health Council of the Netherlands. European primary care [internet].The Hague; 2004 [acesso em 2018 maio 28]. Disponível em: https://www.gezondheidsraad.nl/sites/default/files/European_primary_care_final.pdf.

13. Kringos DS, Boerma WG, Hutchinson A, et al. The breadth of primary care: a systematic literature review of its core dimensions. BMC Health Serv Res [internet]. 2010 [acesso em 2018 maio 28];10(1):65. Disponível em: http://www.ncbi.nlm.nih.gov/pubmed/20226084.

14. Nedel FB, Facchini LA, Martín M, et al. Características da atenção básica associadas ao risco de internar por condições sensíveis à atenção primária: revisão sistemática da literatura. Epidemiol e Serviços Saúde [internet]. 2010 [acesso em 2018 maio 28]; 19(1):61-75. Disponível em: http://scielo.iec.pa.gov. 
br/scielo.php?script=sci_arttext\&pid $=$ S1679$-49742010000100008 \& \operatorname{lng}=$ pt\&nrm=iso\&tlng=pt.

15. Rosenblatt RA, Wright GE, Baldwin LM, et al. The effect of the doctor-patient relationship on emergency department use among the elderly. Am J Public Health [internet]. 2000 [acesso em 2018 maio 28]; 90(1):97-102. Disponível em: http://www.ncbi. nlm.nih.gov/pubmed/10630144.

16. Shi L. Primary Care, Specialty Care, and Life Chances. Int J Heal Serv [internet]. 1994 [acesso em 2018 maio 28]; 24(3):431-58. Disponível em: http://www. ncbi.nlm.nih.gov/pubmed/7928012.

17. Mendes EV. As redes de atenção à saúde. Cienc Saúde Colet. [internet]. 2010 [acesso 2018 maio 28]; 15(5):2297-305. Disppnível: http://www.scielo.br/scielo.php?script=sci_arttext\&pid=S1413$-81232010000500005 \& \operatorname{lng}=$ pt\&tlng=pt.

18. Rodrigues LBB, Silva PCS, Peruhype RC, et al. A atenção primária à saúde na coordenação das redes de atenção: uma revisão integrativa. Ciênc Saúde Colet [internet]. 2014 [acesso em 2018 maio 28];19(2):343-52. Dsponível em: http://www.scielo.br/scielo.php?script=sci_arttext\&pid=S1413$-81232014000200343 \& \operatorname{lng}=$ pt\&tlng=pt.

19. Santos L, Campos GWS. SUS Brasil: a região de saúde como caminho. Saúde e Soc [internet]. 2015 [acesso em 2018 maio 28]; 24(2):438-46. Disponível em: http://www.scielo.br/scielo.php?script=sci_art text\&pid=S0104-12902015000200438\&lng=pt\&tln $\mathrm{g}=\mathrm{pt}$.

20. Almeida PF, Santos AM, Santos VP, et al. Integração assistencial em região de saúde: paradoxo entre necessidades regionais e interesses locais. Saúde e Sociadade [internet]. 2016 [acesso em 2018 maio 28]; 25(2):320-35. Disponível em: http:// www.scielo.br/pdf/sausoc/v25n2/1984-0470-sausoc-25-02-00320.pdf.

21. Sousa MF, Hamann EM. Programa Saúde da Família no Brasil: uma agenda incompleta? Ciênc Saúde Colet. [internet]. 2009 [acesso em 2018 ago 13];
14(Supl 1): 1325-1335. Disponível em: http://www. scielo.br/scielo.php?script=sci_arttext\&pid=S1413-81232009000800002\&lng=en. http://dx.doi. org/10.1590/S1413-81232009000800002.

22. Mendes EV. O cuidado das condições crônicas na atenção primária à saúde: o imperativo da consolidação da estratégia da saúde da família [internet]. Brasília:, DF Organização Pan-Americana da Saúde; 2012 [acesso em 2017 ago 7]. Disponível em: http:// bvsms.saude.gov.br/bvs/publicacoes/cuidado_condicoes_atencao_primaria_saude.pdf.

23. Facchini LA, Silveira DS, Duro SMS, et al. Os sentidos da pesquisa nos processos organizativos da Estratégia Saúde da Família. In: Sousa MF, Franco MS, Mendonça MA, editores. Saúde da família nos municípios brasileiros: os reflexos dos 20 anos no espelho do futuro. Campinas: Saberes; 2014. p. 849-92.

24. Harzheim E, Álvarez-Dardet C. Evaluación de la atención a la salud infantil del Programa Saúde da Família en la región sur de Porto Alegre, Brasil. Rev Bras Med Família e Comunidade [internet]. 2007 [acesso em 2018 maio 28]; 3(9):60. Disponívelm: http://rbmfc.org.br/rbmfc/article/view/84.

25. Macinko J, Almeida C, Oliveira ES. Organization and delivery of primary health care services in Petrópolis, Brazil. Int J Health Plann Manage [internet]. 2004 [acesso em 2018 maio 28]; 19(4):30317. Disponível em: http://doi.wiley.com/10.1002/ hpm.766.

26. Lima-Costa MF, Turci MA, Macinko J. Estratégia Saúde da Família em comparação a outras fontes de atenção: indicadores de uso e qualidade dos serviços de saúde em Belo Horizonte, Minas Gerais, Brasil. Cad Saude Publica [internet]. 2013 [acesso em 2018 maio 28]; 29(7):1370-80. Disponível em: http://www.scielo.br/scielo.php?script=sci arttext\&pid=S0102-311X2013000700011\&lng=pt\&tl ng=pt.

27. Macinko J, Lima Costa MF. Access to, use of and satisfaction with health services among adults enrolled in Brazil's Family Health Strategy: eviden- 
ce from the 2008 National Household Survey. Trop Med Int Heal [internet]. 2012 [acesso em 2018 maio 28]; 17(1):36-42. Disponível em: http://www.ncbi. nlm.nih.gov/pubmed/21859440.

28. van Stralen CJ, Belisário SA, van Stralen TBS, et al. Percepção dos usuários e profissionais de saúde sobre atenção básica: comparação entre unidades com e sem saúde da família na Região Centro-Oeste do Brasil. Cad Saude Pública. 2008; 24(supl):s148-s158.

29. Zils ADA, Castro RCL, Oliveira MMC, et al. Satisfação dos usuários da rede de Atenção Primária de Porto Alegre. Rev Bras Med Família e Comunidade [internet]. 2009 [acesso em 2018 maio 28]; 4(16):270. Disponível em: http://rbmfc.org.br/rb$\mathrm{mfc} /$ article/view/233.

30. Facchini LA, Piccini RX, Tomasi E, et al. Desempenho do PSF no Sul e no Nordeste do Brasil: avaliação institucional e epidemiológica da Atenção Básica à Saúde. Ciênc Saúde Colet. [internet]. 2006 set [acesso em 2018 maio 28]; 11(3):669-81.Disponível em: http://www.scielo.br/scielo.php?script=sci arttext\&pid=S1413-81232006000300015\&lng=pt\&t $\operatorname{lng}=\mathrm{pt}$.

31. Guanais FC. Health equity in Brazil. BMJ [internet]. 2010 [acesso em 2018 maio 28]; 341:c6542. Disponível em: http://www.ncbi.nlm.nih.gov/pubmed/21115590.

32. Thumé E, Facchini LA, Tomasi E, et al. Assistência domiciliar a idosos: fatores associados, características do acesso e do cuidado. Rev. Saúde Públ. [internet]. $2010 \mathrm{dez}$ [acesso em 2018 maio 28]; 44(6):1102-11.Disponível em: http://www.scielo.br/scielo.php?script=sci_arttext $\&$ pid $=$ S0034$-89102010000600016 \& \operatorname{lng}=$ pt\&tlng=pt.

33. Thumé E, Facchini LA, Wyshak G, et al. The Utilization of Home Care by the Elderly in Brazil's Primary Health Care System. Am J Public Health [internet]. 2011 [acesso em 2018 maio 28]; 101(5):868-74. Disponível em: http://www.ncbi.nlm.nih.gov/pubmed/20724683.
34. Brandao JRM, Gianini RJ, Novaes HMD, et al. The family health system: analysis of a health survey in Sao Paulo, Brazil. J Epidemiol Community Heal [internet]. 2011 [acesso em 2018 maio 28]; 65(6):48390. Disponível em: http://www.ncbi.nlm.nih.gov/ pubmed/21148818.

35. Caldeira AP, Oliveira RM, Rodrigues OA. Qualidade da assistência materno-infantil em diferentes modelos de Atenção Primária. Ciênc Saúde Colet. [internet]. 2010 [acesso 2018 maio 28]; 15(supl 2):3139-47. Disponível em: http://www. scielo.br/scielo.php?script=sci_arttext\&pid=S1413$-81232010000800018 \& \operatorname{lng}=$ pt\&tlng=pt.

36. Cesar JA, Sutil AT, Santos GB, et al. Assistência pré-natal nos serviços públicos e privados de saúde: estudo transversal de base populacional em Rio Grande, Rio Grande do Sul, Brasil. Cad Saude Pública [internet]. 2012 [acesso em 2018 maio 28]; 28(11):2106-14. Disponível em: http://www.scielo. br/scielo.php?script=sci_arttext\&pid=S0102-311X2 012001100010\&lng=pt\&tlng=pt.

37. Mendoza-Sassi RA, Cesar JA, Teixeira TP, et al. Diferenças no processo de atenção ao pré-natal entre unidades da Estratégia Saúde da Família e unidades tradicionais em um município da Região Sul do Brasil. Cad Saude Publica [internet]. 2011 [acesso em 2018 maio 28]; 27(4):787-96. Disponível em: http://www.scielo. br/scielo.php?script=sci_arttext\&pid=S0102-311X2011 000400018\&lng=pt\&tlng=pt.

38. Rasella D, Aquino R, Barreto ML. Impact of the Family Health Program on the quality of vital information and reduction of child unattended deaths in Brazil: an ecological longitudinal study. BMC Public Health [internet]. 2010 [acesso em 2018 maio 28]; 10(1):380. Disponível em: http://www.ncbi. nlm.nih.gov/pubmed/20587036.

39. Macinko J, Guanais FC, Fátima M, et al. Evaluation of the impact of the Family Health Program on infant mortality in Brazil, 1990-2002. J Epidemiol Community Heal [internet]. 2006 [acesso em 2018 maio 28]; 60(1):13-9. Acesso em: http://www.ncbi. nlm.nih.gov/pubmed/16361449. 
40. Rocha R, Soares RR. Evaluating the impact of community-based health interventions: evidence from Brazil's Family Health Program. Health Econ [internet]. 2010 [acesso 2018 maio 28]; 19(S1):126-58. Disponível em: http://www.ncbi.nlm.nih.gov/pubmed/20803631.

41. Schmidt MI, Duncan BB, Silva GA, et al. Chronic non-communicable diseases in Brazil: burden and current challenges. Lancet [internet]. 2011 [acesso em 2018 maio 28]; 377(9781):1949-61. Disponível em: http://www.ncbi.nlm.nih.gov/pubmed/21561658.

42. Szwarcwald CL. Strategies for improving the monitoring of vital events in Brazil. Int J Epidemiol [internet]. 2008 ago [acesso em 2018 maio 28]; 37(4):738-44. Disponível em: http://www.ncbi.nlm. nih.gov/pubmed/18653509.

43. Campos AZ, Theme-Filha MM. Internações por condições sensíveis à atenção primária em Campo Grande, Mato Grosso do Sul, Brasil, 2000 a 2009. Cad Saúde Pública [internet]. 2012 [acesso 2018 maio 28]; 28(5):845-55. Disponível em: http://www. scielo.br/scielo.php?script=sci_arttext\&pid=S0102$-311 X 2012000500004 \& \operatorname{lng}=p t \& t \operatorname{lng}=$ pt.

44. Facchini LA, Nobre LCC, Faria NMX, et al. Sistema de Informação em Saúde do Trabalhador: desafios e perspectivas para o SUS. Ciênc Saúde Colet. [internet]. 2005 [acesso em 2018 maio 28]; 10(4):857-67. Disponível em: http://www.scielo.br/scielo.php?script=sci_arttext\&pid=S1413$-81232005000400010 \& \operatorname{lng}=p t \&$ tlng $=$ pt.

45. Mendonca CS, Harzheim E, Duncan BB, et al. Trends in hospitalizations for primary care sensitive conditions following the implementation of Family Health Teams in Belo Horizonte, Brazil. Health Policy Plan [internet]. 2012 [acesso em 2018 maio 28]; 27(4):348-55. Disponível em: http://www.ncbi.nlm. nih.gov/pubmed/21666271.

46. Siqueira FCV, Facchini LA, Silveira DS, et al. Barreiras arquitetônicas a idosos e portadores de deficiência física: um estudo epidemiológico da estrutura física das unidades básicas de saúde em sete estados do Brasil. Ciênc Saúde Colet. [internet]. 2009 fev [acesso em 2018 maio 28]; 14(1):39-44. Disponível em: http://www.scielo.br/scielo.php?script=sci arttext\&pid=S1413-81232009000100009\&lng=pt\&t lng=pt.

47. Souza LL, Costa JSD. Internações por condições sensíveis à atenção primária nas coordenadorias de saúde no RS. Rev. Saúde Pública [internet]. 2011 [acesso em 2018 maio 28]; 45(4):765-72. Disponível em: http://www.scielo.br/scielo.php?script=sci arttext\&pid=S0034-89102011000400017\&lng=pt\&t lng=pt.

48. Norman AH, Tesser CD. Acesso ao cuidado na Estratégia Saúde da Família: equilíbrio entre demanda espontânea e prevenção/promoção da saúde. Saúde e Soc. [internet]. 2015 [acesso em 2018 maio 28]; 24(1):165-79. Disponível em: http://www.scielo.br/scielo.php?script=sci_arttext\&pid=S0104$-12902015000100165 \& \operatorname{lng}=$ pt\&tlng=pt.

49. Tesser CD, Norman AH. Repensando o acesso ao cuidado na Estratégia Saúde da Família. Saúde e Soc [internet]. 2014 set [acesso em 2016 abr 27]; 23(3):869-83. Disponível em: http://www.scielo.br/scielo.php?script=sci_arttext $\&$ pid $=$ S0104$-12902014000300869 \& \operatorname{lng}=$ en $\& n r m=i s o \& t \operatorname{lng}=p t$.

50. Starfield B. Atenção primária: equilibrio entre necessidade de saúde, serviços e tecnologia. 1. ed. Werthein J, Braslavsky C, Tedesco JC, et al., editores. Brasília: UNESCO; Ministério da Saúde; 2002.

51. Assis MMA, Jesus WLA. Acesso aos serviços de saúde: abordagens, conceitos, políticas e modelo de análise. Ciênc Saúde Colet. [internet]. 2012 [acesso em 2018 maio 28]; 17(11):2865-75. Disponível em: http://www.scielo.br/scielo.php?script=sci arttext\&pid=S1413-81232012001100002\&lng=pt\&tl ng=pt.

52. Brasil. Ministério da Saúde . HumanizaSUS: Política Nacional de Humanização: a humanização como eixo norteador das práticas de atenção e gestão em todas as instâncias do SUS. [internet]. Brasília, DF: 
MS; 2004 [acesso em 2018 maio 28]. Disponível em: http://bvsms.saude.gov.br/bvs/publicacoes/humanizasus_2004.pdf.

53. Franco TB, Bueno WS, Merhy EE. O acolhimento e os processos de trabalho em saúde: o caso de Betim, Minas Gerais, Brasil. Cad Saude Publica [internet]. 1999 abr [acesso em 2018 maio 28]; 15(2):34553. Disponível em: http://www.scielo.br/scielo. php?script=sci_arttext\&pid=S0102-311X19990002 00019\&lng=pt\&tlng=pt.

54. Mitre SM, Andrade EIG, Cotta RMM. Avanços e desafios do acolhimento na operacionalização e qualificação do Sistema Único de Saúde na Atenção Primária: um resgate da produção bibliográfica do Brasil. Ciênc Saúde Colet. [internet]. 2012 [acesso em 2018 maio 28]; 17(8):2071-85. Disponível em: http://www.scielo.br/scielo.php?script=sci arttext\&pid=S1413-81232012000800018\&lng=pt\&tl ng=pt.

55. Souza ECF, Vilar RLA, Rocha NSPD, et al. Acesso e acolhimento na atenção básica: uma análise da percepção dos usuários e profissionais de saúde. Cad Saude Publica [internet]. 2008 [acesso em 2018 maio 28]; 24(supl 1):s100-10. Disponível em: http://www.scielo.br/scielo.php?script=sci arttext\&pid=S0102-311X2008001300015\&lng=pt\&t $\operatorname{lng}=\mathrm{pt}$.

56. Tesser CD, Poli Neto P, Campos GWS. Acolhimento e (des)medicalização social: um desafio para as equipes de saúde da família. Ciênc Saúde Colet. [internet]. 2010 nov [acesso em 2018 maio 28]; 15(supl 3):3615-24. Disponível em: http://www. scielo.br/scielo.php?script=sci_arttext\&pid=S1413$-81232010000900036 \& \operatorname{lng}=$ pt\&tlng=pt.

57. Schimith MD, Lima MADS. Acolhimento e vínculo em uma equipe do Programa Saúde da Família. Cad Saude Publica [internet]. 2004 dez [acesso em 2018 maio 28]; 20(6):1487-94. Disponível em: http://www.scielo.br/scielo.php?script=sci_ arttext\&pid=S0102-311X2004000600005\&lng=pt\& tlng=pt.
58. Cavalcante Filho JB, Vasconcelos EMS, Ceccim RB, et al. Acolhimento coletivo: um desafio instituinte de novas formas de produzir o cuidado. Interface - Comun Saúde, Educ [internet]. 2009 [acesso em 2018 maio 28]; 13(31):315-28. Disponível em: http://www.scielo.br/scielo.php?script=sci arttext\&pid=S1414-32832009000400007\&lng=pt\& tlng=pt.

59. Viacava F, Bellido JG. Health, access to services and sources of payment, according to household surveys. Ciênc saúde coletiva [internet]. $2016 \mathrm{fev}$ [acesso em 2018 ago 12]; 21(2): 351-370. Disponível em: http://www.scielo. $\mathrm{br} /$ scielo.php? script $=$ sci_arttext $\&$ pid $=$ S1413$-81232016000200351 \& \operatorname{lng}=\mathrm{en}$. http://dx.doi. org/10.1590/1413-81232015212.19422015.

60. Brasil. Ministério da Saúde. Histórico de Cobertura da Saúde da Família no Brasil [internet]. Brasília, DF: MS; 2017 [acesso em 2017 ago 21]. Disponível em: http://dab.saude.gov.br/portaldab/historico_ cobertura_sf.php.

61. Bousquat A, Giovanella L, Fausto MCR, et al. Tipologia da estrutura das unidades básicas de saúde brasileiras: os 5 R. Cad Saúde Pública [internet]. 2017 [acesso em 2018 ago 12]; 33(8):e00037316. Disponível em: http://www.scielo.br/scielo.php?script=sci_ arttext\&pid=S0102-311X2017000805005\&lng=en.

62. Teixeira CF, Sola JP. Modelo de atenção à saúde no SUS: trajetória do debate conceitual, situação atual, desafios e perspectiva [internet]. 3. ed. Salvador: UFBA; 2006 [acesso em 2018 maio 28]. Disponível em: http://books.scielo.org/id/f7/pdf/ teixeira-9788523209209-07.pdf

63. Sousa MF. O Programa Saúde da Família no Brasil: análise do acesso à atenção básica. Rev Bras Enferm [internet]. 2008 [acesso em 2018 maio 28]; 61(2):153-8. Disponível em: http://www.scielo.br/scielo.php?script=sci_arttext $\&$ pid=S0034$-71672008000200002 \& \operatorname{lng}=$ pt\&tlng=pt.

64. Silva LA, Casotti CA, Chaves SCL. A produção científica brasileira sobre a Estratégia Saúde da Famí- 
lia e a mudança no modelo de atenção. Ciênc Saúde Colet. [internet]. 2013 jan [acesso em 2018 maio 28]; 18(1):221-32. Disponível em: http://www.scielo.br/scielo.php?script=sci_arttext\&pid=S1413$-81232013000100023 \& \operatorname{lng}=$ pt\&tlng=pt.

65. Azevedo ALM, Costa AM. A estreita porta de entrada do Sistema Único de Saúde (SUS): uma avaliação do acesso na Estratégia de Saúde da Família. Interface - Comun Saúde, Educ [internet]. 2010 [acesso em 2018 maio 28]; 14(35):797-810. Disponível em: http://www.scielo.br/scielo.php?script=sci_ arttext\&pid=S1414-32832010000400007\&lng=pt\&t lng=pt.

66. Luz J. Implantação do processo de trabalho da Estratégia Saúde da Família em Florianópolis. Florianópolis: UFSC; 2009.

67. Barbosa SP. Acesso à atenção primária à saúde em municípios de uma região de saúde de Minas Gerais [tese] [internet]. Belo Horizonte: Universidade Federal de Minas Gerais; 2013. 134 p. [acesso em 2018 maio 28]. Disponível em: http://www.enf.ufmg.br/ pos/defesas/507D.PDF.

68. Silva SA, Baitelo TC, Fracolli LA. Avaliação da Atenção Primária à Saúde: a visão de usuários e profissionais sobre a Estratégia de Saúde da Família. Rev Lat Am Enfermagem [internet]. 2015 [acesso em 2018 maio 28]; 23(5):979-87. Disponível em: http://www.scielo.br/pdf/rlae/v23n5/pt_01041169-rlae-23-05-00979.pdf.

69. Marin MJS, Marchioli M, Moracvick MYAD. Fortalezas e fragilidades do atendimento nas unidades básicas de saúde tradicionais e da estratégia de saúde da família pela ótica dos usuários 1 strengths and weaknesses of the care delivered in the traditional primary healthcare units and family healthcare strategy units in the perspective of users. Texto Context Enferm [internet]. 2013 [acesso em 2018 maio 28]; 22(3):780-8. Disponível em: http://www. scielo.br/pdf/tce/v22n3/v22n3a26.pdf.

70. Sisson MC, Andrade SR, Giovanella L, et al. Estratégia de Saúde da Família em Florianópolis: inte- gração, coordenação e posição na rede assistencial. Saúde e Soc. 2011; 20(4):991-1004.

71. Paula WKAS, Samico IC, Caminha MFC, et al. Primary health care assessment from the users' perspectives: a systematic review. Rev da Esc Enferm da USP [internet]. 2016 [acesso em 2018 maio 28]; 50(2):335-45. Disponível em: http://www.scielo.br/scielo.php?script=sci_arttext $\&$ pid $=$ S0080$-62342016000200335 \& \operatorname{lng}=$ en\&tlng=en.

72. Arruda CAM, Bosi MLM. Satisfação de usuários da atenção primária à saúde: um estudo qualitativo no Nordeste do Brasil. Interface [internet]. 2016 [acesso em 2018 maio 28]; 21(61):321-32. Disponível em: http://www.scielo.br/scielo.php?script=sci arttext\&pid=S1414-32832017000200321\&lng=pt\&tl $\mathrm{ng}=\mathrm{pt}$.

73. Gomes MCPA, Pinheiro R. Acolhimento e vínculo: práticas de integralidade na gestão do cuidado em saúde em grandes centros urbanos. Interface [internet]. 2005 [acesso em 2018 maio 28]; 9(17):287-301. Disponível em: http://www.scielo.br/scielo.php?script=sci_arttext\&pid=S1414$-32832005000200006 \& \operatorname{lng}=$ pt\&tlng=pt.

74. Harzheim E, Oliveira MMC, Agostinho MR, et al. Validação do instrumento de avaliação da atenção primária à saúde: PCATool-Brasil adultos. Rev Bras Med Família e Comunidade. 2013; 8(29):274-84.

75. Macinko J, Almeida C, Sa PK. A rapid assessment methodology for the evaluation of primary care organization and performance in Brazil. Health Policy Plan [internet]. 2007 [acesso em 2018 maio 28]; 22(3):167-77. Disponível em: http://www.ncbi.nlm. nih.gov/pubmed/17400576.

76. van Stralen CJ, Belisário SA, van Stralen TBS, et al. Percepção dos usuários e profissionais de saúde sobre atenção básica: comparação entre unidades com e sem saúde da família na Região Centro-Oeste do Brasil. Cad Saude Publica [internet]. 2008 [acesso em 2018 maio 28]; 24(supl 1):s148-58. Disponível em: http://www. scielo.br/scielo.php?script=sci_arttext\&pid=S0102$-311 X 2008001300019 \& \operatorname{lng}=p t \& t \operatorname{lng}=p t$. 
77. Castro RCL, Knauth DR, Harzheim E, et al. Avaliação da qualidade da atenção primária pelos profissionais de saúde: comparação entre diferentes tipos de serviços. Cad Saude Publica [internet]. 2012 set [acesso em 2018 maio 28]; 28(9):1772-84. Disponível em: http://www. scielo.br/scielo.php?script=sci_arttext\&pid=S0102$-311 X 2012000900015 \& \operatorname{lng}=p t \& t \operatorname{lng}=p t$.

78. Cesar MC, Campos GWS, Montebelo MIL, et al.Avaliação da atenção primária no município de Piracicaba, São Paulo, Brasil. Saúde debate [internet]. 2014 [acesso em 2018 maio 28]; 38(esp):296306. Disponível em: http://www.gnresearch.org/ doi/10.5935/0103-1104.2014S022.

79. Ferreira VD, Oliveira JM, Maia MAC, et al. Assessment of Primary Healthcare attributes in one Municipality of Minas Gerais State. Esc. Anna Nery Rev. Enferm. [internet]. 2016 [acesso em 2018 maio 28]; 20(4). Disponível em: http://www.gnresearch.org/ doi/10.5935/1414-8145.20160104.

80. Chomatas E, Vigo A, Marty I, et al. Avaliação da presença e extensão dos atributos da atenção primária em Curitiba. Rev Bras Med Família e Comunidade. 2013; 8(29):294-303.

81. Paula FA, Silva CCR, Santos DF, et al. Avaliação da atenção à saúde do adulto em um município-polo do Vale do Jequitinhonha (MG). Saúde debate [internet]. 2015 [acesso em 2016 jul 19]; 39(106):80214. Disponível em: http://www.scielo.br/pdf/sdeb/ v39n106/0103-1104-sdeb-39-106-00802.pdf.

82. Alves MGM, Casotti E, Oliveira LGD, et al. Fatores condicionantes do acesso às equipes da Estratégia Saúde da Família no Brasil. Saúde debate [internet]. 2014 [acesso em 2018 maio 28]; 38(esp):34-51. Disponível em: http://www.gnresearch.org/ doi/10.5935/0103-1104.2014S004.

83. Uchôa SAC, Arcêncio RA, Fronteira I, et al. Acesso potencial à Atenção Primária à Saúde: o que mostram os dados do Programa de Melhoria do Acesso e da Qualidade do Brasil? Rev Lat Am Enfermagem [internet]. 2016 [acesso em 2018 maio 28]; 24(e2672):30. Disponível em: www.eerp.usp.br/rlae.
84. Conill EM. Ensaio histórico-conceitual sobre a Atenção Primária à Saúde: desafi os para a organização de serviços básicos e da Estratégia Saúde da Família em centros urbanos no Brasil A historical and conceptual model for Primary Health Care: challenges for the organization of primary care and the Family Health Strategy in large Brazilian cities. Cad Saude Publica [internet]. 2008 [acesso em 2018 maio 28]; 24(1):7-27. Disponível em: http://www. scielo.br/pdf/csp/v24sl/02.pdf.

85. Campos RTO, Ferrer AL, Gama CAP, et al. Avaliação da qualidade do acesso na atenção primária de uma grande cidade brasileira na perspectiva dos usuarios. Saúde debate [internet]. 2014 [acesso em 2018 maio 28]; 38(special):252-64. Disponível em: http://www.gnresearch.org/doi/10.5935/0103-1104.2014 S019.

86. The NHS Plan: a plan for investment, a plan for reform [internet]. 2000. [acesso em 2018 ago 12]. Disponível em: http://webarchive.nationalarchives. gov.uk/20130124064356/http://www.dh.gov.uk/ prod_consum_dh/groups/dh_digitalassets/@dh/@ en/@ps/documents/digitalasset/dh_118522.pdf.

87. Heath I. In defence of a National Sickness Service. BMJ [internet]. 2007 jan 6 [acesso em 2018 maio 28]; 334(7583):19. Disponível em: http://www.ncbi. nlm.nih.gov/pubmed/17204798.

88. Starfield B, Hyde J, Gervas J, et al. The concept of prevention: a good idea gone astray? J Epidemiol Community Heal [internet]. 2008 [acesso em 2018 maio 28]; 62(7):580-3. Disponível em: http://www. ncbi.nlm.nih.gov/pubmed/18559439.

89. Gérvas J, Starfield B, Heath I. Is clinical prevention better than cure? Lancet [internet]. 2008 [acesso em 2018 maio 28]; 372(9654):1997-9. Disponível em: http://www.ncbi.nlm.nih.gov/pubmed/19059049.

90. Tesser CD. Por que é importante a prevenção quaternária na prevenção? Rev Saude Publica [internet]. 2017 [acesso em 2018 maio 28]; 51:116. Disponível em: https://www.revistas.usp.br/rsp/article/ view/141548. 
91. Ilic D, Neuberger MM, Djulbegovic M, et al. Screening for prostate cancer. Cochrane Database Syst Rev [internet]. 2013 [acesso em 2018 ago 10]; (1):CD004720. Disponível em: http://www.ncbi. nlm.nih.gov/pubmed/23440794.

92. Tesser CD, d'Ávila TLC. Por que reconsiderar a indicação do rastreamento do câncer de mama? Cad Saude Pública [internet]. 2016 [acesso em 2018 ago 10]; 32(5). Disponível em: http://www.scielo.br/ scielo.php?script=sci_arttext\&pid=S0102-311X2016 $000500706 \& \operatorname{lng}=p t \& \ln g=p t$.

93. Brasil. Ministério da Saúde. Cadernos de Atenção Primária. Rastreamento [internet]. Brasília, DF: MS; 2010 [acesso em 2018 ago 10]. Disponível em: http://bvsms.saude.gov.br/bvs/publicacoes/caderno_atencao_primaria_29_rastreamento.pdf.

94. Krogsbøll LT, Jørgensen KJ, Grønhøj L C, et al. General health checks in adults for reducing morbidity and mortality from disease: Cochrane systematic review and meta-analysis. BMJ [internet]. 2012 [acesso em 2018 ago 10]; 345:e7191. Disponível em: http://www.ncbi.nlm.nih.gov/pubmed/23169868.

95. Fontana KC, Lacerda JT, Machado PM. O processo de trabalho na Atenção Básica à saúde: avaliação da gestão. Saúde em Debate [internet]. 2016 [acesso em 2018 maio 28]; 40(110):64-80. Disponível em: http://www. scielo.br/scielo.php?script=sci_arttext\&pid=S0103$-11042016000300064 \& \operatorname{lng}=p t \& t \operatorname{lng}=$ pt.

96. Oliveira CVS, Coriolano-Marinus MWL. Desafios do acolhimento na estratégia saúde da família: uma revisão integrativa. Saúde em Redes [internet]. 2016 [acesso em 2018 maio 28]; 2(2):211-25. Disponível em: http://revista.redeunida.org.br/ojs/index.php/ rede-unida/article/view/678.

97. Nobre JPS, Vieira JPA, Gadelha RRM, et al. A percepção dos trabalhadores de saúde sobre o acolhimento no contexto da atenção básica. Rev Expressão Católica Saúde [internet]. 2016 [acesso em 2018 maio 28];1(1). Disponível em: http://publicacoesacademicas.fcrs.edu.br/index.php/recsaude/article/ view/1364.
98. Lopes AS, Vilar RLA, Melo RHV, et al. O acolhimento na Atenção Básica em saúde: relações de reciprocidade entre trabalhadores e usuários. Saúde debate [internet]. 2015 [acesso em 2018 maio 28]; 39(104):114-23. Disponível em: http://www.scielo.br/scielo.php?script=sci_arttext $\&$ pid $=$ S0103$-11042015000100114 \& \operatorname{lng}=p t \& \operatorname{lng}=p t$.

99. Agousto DK, David L, Oliveira DOPS, et al. Da Trindade TG, Lermen Junior N, Poli Neto P. Quantos médicos de família e comunidade temos no Brasil? Rev Bras Med Família e Comunidade [internet]. 2018 [acesso em 2018 ago 10]; 13(40):1-4. Disponível em: https://www.rbmfc.org.br/rbmfc/article/ view/1695.

100.Scheffer M, coordenador. Demografia Médica no Brasil 2018. São Paulo: Departamento de Medicina Preventiva da Faculdade de Medicina da USP; Conselho Regional de Medicina do Estado de São Paulo; Conselho Federal de Medicina; 2018. [acesso em 2018 ago 12]. Disponível em: https://jornal.usp.br/ wp-content/uploads/DemografiaMedica2018.pdf.

101. Boyle S. United Kingdom (England): Health system review. Health Syst Transit [internet]. 2011 [acesso em 2018 maio 28]; 13(1):1-483. Disponível em: http://www.ncbi.nlm.nih.gov/pubmed/21454148.

102. Soares A, Santos NR. Funding of the Unified Health System in FHC, Lula and Dilma government. Saúde debate [internet]. 2014 [acesso em 2018 maio 28]; 38(100):18-25. Disponível em: http://www.gnresearch.org/doi/10.5935/0103-104.20140002.

103. Marques RM, Piola SF, Roa AC. Sistema de Saúde no Brasil: organização e financiamento. Rio de Janeiro: MS; 2016.

104.Brasil. Ministério da Saúde. Portaria no 2.436, de 21 de setembro de 2017. Aprova a Política Nacional de Atenção Básica, estabelecendo a revisão de diretrizes para a organização da Atenção Básica, no âmbito do Sistema Único de Saúde (SUS). Diário Oficial da União. 22 Dez 2017. [acesso em 2018 out 3]. Disponível em: https://www.nescon.medicina.ufmg. br/biblioteca/imagem/portaria2436.pdf. 
105. Green LA, Fryer GE, Yawn BP, et al. The Ecology of Medical Care Revisited. N Engl J Med [internet]. 2001 [acesso em 2018 maio 28]; 344(26):2021-5. Disponível em: http://www.ncbi.nlm.nih.gov/pubmed/11430334.

106.Giovanella L, Escorel S, Mendonça MHM. Estudo de Caso sobre Implementação da Estratégia Saúde da Família em Quatro Grandes Centros Urbanos. Relatório final: Florianópolis [internet]. Rio de Janeiro: Fiocruz; MS; 2009. [acesso em 2017 ago 21]. Disponível em: http://www.pucsp.br/prosaude/downloads/bibliografia/Avaliacao_ESF_Florianopolis.pdf.

107. Masseria C, Irwin R, Thomson S, et al. Primary care in Europe. The london school of economics and political science. Londres: London School; 2009.

108. Gérvas J, Pérez Fernández M. Sano y salvo (y libre de intervenciones médicas innecesarias) [internet]. Barcelona: Los Libros del Lince; 2013 [acesso em 2018 maio 28]. Disponível em: http://equipocesca. org/sano-y-salvo-y-libre-de-intervenciones-medicas-innecesarias-2/.

109. Brasil. Ministério da Saúde. Lei no 12.871, de 22 de outubro de 2013 [internet]. Institui o Programa Mais Médicos, altera as Leis no 8.745, de 9 de dezembro de 1993, e no 6.932, de 7 de julho de 1981, e dá outras providências. Diário Oficial da União. 22 Out. 2013 [acesso em 2018 maio 28]. Disponível em: http://www.planalto.gov.br/ccivil_03/_Ato20112014/2013/Lei/L12871.htm

110. Campos GWDS. Um metodo para analise e co-gestao de coletivos. 2 ed. São Paulo: Hucitec; 2015.

111. Poli Neto P, Tesser CD, Monteiro GH, et al. O uso do correio eletrônico na comunicação entre usuários e uma equipe de saúde da família: relato de experiência. Rev Bras Med Família e Comunidade [internet]. 2015 [acesso em 2018 maio 28]; 10(37):1. Disponível em: https://rbmfc.org.br/rbmfc/article/view/1167.

112. Sousa LD, Lunardi Filho WD, Lunardi VL, et al. A produção científica de enfermagem acerca da clíni- ca: uma revisão integrativa. Rev da Esc Enferm da USP [internet]. 2011 abr [acesso em 2018 maio 28]; 45(2):494-500. Disponível em: http://www.scielo.br/scielo.php?script=sci_arttext $\&$ pid $=$ S0080$-62342011000200027 \& \operatorname{lng}=$ pt\&tlng=pt.

113. Murray M, Tantau C. Same-day appointments: exploding the access paradigm. Fam Pract Manag [internet]. 2000 set [acesso em 2017 ago 18]; 7(8):4550. Disponível em: http://www.ncbi.nlm.nih.gov/ pubmed/11183460.

114. Murray M, Berwick DM. Advanced access: reducing waiting and delays in primary care. JAMA [internet]. 2003 [acesso em $2018 \mathrm{fev} 28$ ]; 289(8):103540. Disponível em: http://www.ncbi.nlm.nih.gov/ pubmed/12597760.

115. Norman AH, Tesser CD. Prevenção quaternária: as bases para sua operacionalização na relação médico-paciente. Rev Bras Med Família e Comunidade [internet]. 2015 [acesso em 2018 maio 28]; 10(35):1. Disponível em: https://rbmfc.org.br/rbmfc/article/ view/1011.

116. Gómez Santana MDC, Gavilán-Moral E, Villafaina-Barroso A, et al. Prescripción prudente y deprescripción de fármacos como herramientas para la prevención cuaternaria. Rev Bras Med Família e Comunidade [internet]. 2015 [acesso em 2018 maio 28]; 10(35):1. Disponível em: https://rbmfc.org.br/ rbmfc/article/view/1021.

117. Costa-Rosa A da. Atenção Psicossocial além da Reforma Psiquiátrica Abílio da Costa-Rosa Contribuições a uma Clínica Crítica dos processos de subjetivação na Saúde Coletiva [internet]. São Paulo: Universidade Estadual Paulista; 2013 [acesso em 2018 ago 9]. 337 p. Disponível em: http://medicalizacao.org.br/wp-content/uploads/2014/08/0.-Costa-Rosa-A.-Atenção-Psicossocial-além-da-Reforma-Psiquiátrica-versão-revisada.pdf.

118. Frosi RV, Tesser CD. Práticas assistenciais em saúde mental na atenção primária à saúde: análise a partir de experiências desenvolvidas em Florianópolis, Brasil. Ciênc Saúde Colet. [internet]. 2015 
[acesso em 2018 maio 28]; 20(10):3151-61. Disponível em: http://www.scielo.br/scielo.php?script=sci arttext\&pid=S1413-81232015001003151\&lng=pt\&tln $\mathrm{g}=\mathrm{pt}$.

119. Tesser CD, Sousa IMC. Atenção primária, atenção psicossocial, práticas integrativas e complementares e suas afinidades eletivas. Saúde e Soc [internet]. 2012 [acesso em 2018 maio 28]; 21(2):336-50. Disponível em: http://www.scielo.br/scielo.php?script=sci_arttext\&pid=S0104$-12902012000200008 \& \operatorname{lng}=$ pt\&tlng=pt.

120. World Health Organization. WHO Traditional Medicine Strategy 2014-2023 [internet]. Hong Kong; 2013 [acesso em 2018 ago 9]. Disponível em: www. who.int.

121. Tesser CD, d'Ávila TLC, Tesser CD, et al. É necessário mudar a perspectiva sobre o câncer de mama: afastar o rastreamento e priorizar a agilidade no diagnóstico e tratamento. Cad Saude Pública [inter- net]. 2016 [acesso em 2018 ago 9]; 32(5). Disponível em: http://www.scielo.br/scielo.php?script=sci arttext\&pid=S0102-311X2016000500708\&lng=pt\& tlng=pt.

122. Tesser CD, Norman AH. Differentiating clinical care from disease prevention: a prerequisite for practicing quaternary prevention. Cad Saude Publica [internet]. 2016 [acesso em 2018 maio 28]; 32(10):e00012316. Disponível em: http://www.ncbi. nlm.nih.gov/pubmed/27783750.

123. Vidal TB, Tesser CD, Harzheim E, et al. Modelos de agendamento e qualidade da atenção primária: estudo transversal multinível. Rev Saude Pública. 2018 (no prelo).

Recebido em 30/05/2018

Aprovado em 17/08/2018

Conflito de interesses: inexistente

Suporte financeiro: não houve 